\title{
The nominative technique: a simple tool for assessing illegal wildlife consumption
}

\author{
Elizabeth Oneita Davis, Brian Crudge and Jenny Anne Glikman
}

\begin{abstract}
The aim of our study was to test the efficacy of the nominative technique for estimating the prevalence of wildlife part use within a small sample. We used the domestic consumption of bear Ursus thibetanus and Helarctos malayanus parts in Lao People's Democratic Republic (Laos) as a case study and performed 179 semi-structured interviews in Luang Prabang, northern Laos, in August 2017 and April 2019. We also assessed whether the specialized questioning of the nominative technique could be used for qualitative data collection methods, such as semi-structured interviews. The technique theoretically ensures more accurate statements of illegal wildlife consumption by maintaining the anonymity of an individual's sensitive behaviour through asking about the behaviour of peers. We also directly asked about participants' use of bear parts. The nominative technique suggested that c. $11 \%$ of the participants' peers used bear parts, whereas respondents' direct admittance of using bear parts was approximately double, at $23 \%$. Use of bear parts appears not to be sensitive in northern Laos. In addition, we found a strong association between responses to questioning using the nominative technique and direct questioning, indicating that users of bear parts have social networks with higher levels of use. This lends supports to theories that use of wildlife products is directly influenced by social group. The underreporting resulting from use of the nominative technique indicates the high variability of response that can occur within small samples. However, our results show that the nominative technique may be a simple, useful tool for triangulating data, assessing users' integration into social networks of use, and assessing changes in behaviour prevalence.
\end{abstract}

Keywords Bear bile, illegal wildlife trade, Lao PDR, nominative technique, specialized questioning technique, Ursus thibetanus
Supplementary material for this article is available at doi.org/10.1017/So030605320000745

D esearch on wildlife trade requires frequent adaptation Rand innovation to match the constantly evolving legal and illegal system of this trade and of consumption. As a simple example, online traders of illegal wildlife regularly change the keywords they use, to avoid detection, thus obfuscating attempts to identify and halt online trade (Hernandez-Castro \& Roberts, 2015). In addition, bias can occur in estimates of consumption prevalence, primarily because of respondents' concerns over illegality, and/or concerns about the social undesirability of the behaviour (St John et al., 2010; Nuno \& St John, 2015).

Surveying individuals to document the prevalence of a behaviour and identify conservation priorities requires innovative techniques to overcome sensitivity regarding admittance of wildlife consumption. We used the underutilized nominative technique to gain an understanding of the baseline prevalence of an illegal wildlife consumption behaviour: bear part use. Asiatic black bear Ursus thibetanus and, to a lesser extent, sun bear Helarctos malayanus parts and products are known to be in demand and traded in our study site of Luang Prabang, northern Lao People's Democratic Republic (Laos) (Davis et al., 2016; Davis \& Glikman, 2020; Fig. 1). Luang Prabang is the formal royal capital of Lao PDR, a UNESCO World Heritage Site and spiritual centre of Buddhism, and consequently is a popular destination for tourism.

The nominative technique was initially used successfully in North America as a means of investigating heroin use (Miller, 1985). It was adapted for use in conservation to investigate fishing non-compliance in Wales (St John et al., 2010). The nominative technique has been used to investigate use of bear parts in Cambodia (Davis et al., 2019a). It was found to be useful because it had little statistical variability compared to other specialized questioning techniques such as the unmatched count technique; however, it was more sensitive to inaccuracy if an interview team was not well-trained in its application. The use of the nominative technique is relevant for a behaviour such as bear part use that is an illegal activity in Laos (Davis et al., 2016). Although most specialized questioning techniques require large sample sizes, to mitigate statistical error (Nuno \& St John, 2015), smaller samples may suffice for the nominative technique to
Elizabeth Oneita Davis (Corresponding author, (iD) orcid.org/0000-0003-34464471) and Jenny Anne Glikman* (D) orcid.org/0000-0002-0208-5488) San Diego Zoo, 15600 San Pasqual Valley Road, Escondido, California 92026, USA E-mail edavis@sandiegozoo.org

Brian CRUdge (1D orcid.org/0000-0001-9578-6592) Free the Bears, Luang Prabang, Lao PDR

${ }^{*}$ Also at: Instituto de Estudios Sociales Avanzados, Cordoba, Spain

Received 30 April 2020. Revision requested 28 July 2020.

Accepted 30 July 2020. First published online 2 December 2020.

This is an Open Access article, distributed under the terms of the Creative Commons Attribution-NonCommercial-ShareAlike licence (http://creativecommons.org/licenses/by-nc-sa/4.0/), which permits non-commercial re-use, distribution, and reproduction in any medium, provided the same Creative Commons licence is included and the original work is properly cited. 


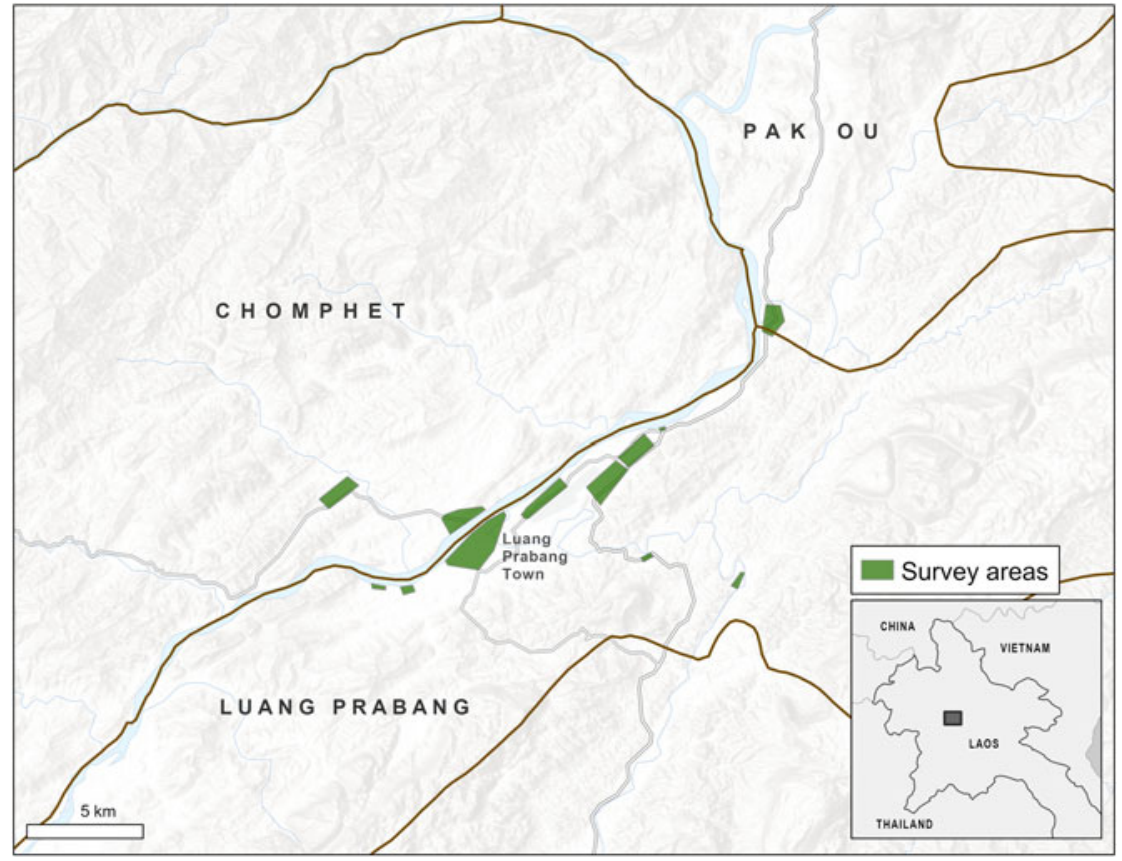

FIG. 1 The Luang Prabang District and town of northern Laos, with survey areas indicated in green, and District borders. Our surveys took place in Luang Prabang, Chomphet and Pak Ou Districts. be valid. Miller (1985) collected data on samples of $>1,000$ respondents and found estimates to be precise. However, the smaller sample studies of St John et al. (2010) (58) and Davis et al. (2019a) (c. 60o) noted that imprecise estimates were a result of lack of knowledge about one's peers, and imprecise questioning within the data collection, respectively, rather than statistical noise. Generally, large samples are needed to overcome the statistical noise that occurs with other specialized questioning techniques (e.g. for the unmatched count technique; Hinsley et al., 2019). Therefore, theoretically there is potential for the nominative technique to perform effectively with sample sizes of $<1,000$. A specialized questioning technique that is robust with a small sample size would be a useful tool for scientists assessing behaviour prevalence. Additionally, unlike many other specialized questioning techniques (e.g. Nuno \& St John, 2015; Bova et al., 2018), the nominative technique uniquely interrogates an individual's social group, which provides additional insights into the prevalence of a behaviour within a community.

Our principal aims were to test the efficacy of the nominative technique when sample sizes are small, and to test whether the technique can be used within semi-structured interviews (Supplementary Material 1). We conducted a total of 179 semi-structured interviews in Luang Prabang, with 79 in August 2017 and 100 in April 2019. All interviews were conducted by EOD and executed by an interpreter in the Lao language. We used convenience sampling, approaching people in public areas such as markets and the street, and interviews alternated between male and female respondents. Potential interviewees were informed that the survey was completely confidential and anonymous, and that they could refuse to answer any question or stop the interview at any time. Free, prior and informed verbal consent was obtained from all participants.

We asked several initial questions related to health and bear bile consumption. We then asked questions regarding prevalence, using the nominative technique, and then questioned people directly. The first question using the nominative technique was: 'How many of your close friends do you know for certain have used/consumed bear parts for medicinal or other purposes?' If the response was a number $\geq 1$, the respondent was then asked to consider that/those friend(s) and nominate one friend from that group. The interviewer then asked the follow-up question 'Other than you, how many people do you believe know that the nominated friend has used bear parts or products for medicinal or other purposes?' For each question, the respondent gave a number. The follow-up question accounts for duplication in which multiple respondents report the same individual. The recall period was not defined.

Following these nominative technique questions, we asked individuals 'Have you ever consumed bear parts?'

The results from the nominative technique were analysed using:

$$
T_{\mathrm{X}}=\sum_{\mathrm{j}=1}^{\mathrm{n}} \frac{A_{\mathrm{j}}}{1+B_{\mathrm{j}}}
$$

(Nuno \& St John, 2015), where $T_{\mathrm{X}}$ is the true proportion of individuals in a sample $(n)$ performing the sensitive behaviour, $A_{j}$ is the number of rule breakers that $j$ knows, and $B_{j}$ is the number of friends, other than $j$, who know of the nominated friend's rule-breaking (Nuno \& St John, 2015). We calculated 
standard errors based on the distribution of individual, calculated proportions. Pearson's correlation coefficient, calculated using the package cor in R 4.0.2 (R Core Team, 2018), was used to investigate whether there was any correlation between the individual direct question responses and the individual calculated nominative technique proportions, and thus to assess the internal consistency of the nominative technique.

The per cent of individuals who directly stated they had used bear parts was $23.5 \pm \mathrm{SE} 3.2 \%$. An estimate of $11.3 \pm \mathrm{SE}$ $2.8 \%$ was obtained with the nominative technique from the calculated proportion of individuals who had used bear parts $\left(T_{\mathrm{X}}\right)$. There was a high correlation between the calculated nominative technique proportion estimate for each individual compared with their responses to direct questioning $(R=0.73, \mathrm{P}<0.05$, CI $0.67-0.79)$.

The lower estimate of bear part use from the nominative technique compared to that estimated from direct questioning suggested bear part use is not widely considered to be a sensitive topic in Luang Prabang, probably because of a lack of enforcement of laws regarding illegal trade and consumption (e.g. Gomez \& Shepherd, 2018). The lower estimate from the nominative technique could be a reflection of individuals knowing relatively few close friends and family who used bear parts and products $\left(A_{j}\right.$ in equation (1)), but reporting high numbers of acquaintances who were aware of use by a friend or family member $\left(B_{j}\right)$ because of the openness of the use of bear parts. Nevertheless, the nominative technique performed well in that the estimate was sensible (i.e. not negative or zero), both within this small sample size and within the qualitative semi-structured interviews.

The high correlation between results from the nominative technique and direct questioning indicates a relationship between admittance of use and a high likelihood of being within a network of individuals who use bear products. It is possible that, as in Viet Nam (Davis et al., 2019b), the social group can be a motivator to begin and/or maintain consumption of bear parts or products.

The correlation analysis suggested that deceit was unlikely to have been acting within the sample. With deceit, we would have expected greater inconsistency between an individual's responses to direct questioning and to the nominative technique questions. The nominative technique appears to be a useful triangulation method for situations in which the sensitivity of a behaviour is unknown. Because of the simplicity of execution, it would be beneficial to include the nominative technique in any study using direct questions to investigate a sensitive behaviour.

Although specialized questioning techniques appear to be useful for accurately estimating the prevalence of sensitive behaviours, there are still problems with implementation of the techniques and a lack of clarity around the appropriateness of the techniques in certain contexts (Chuang et al., 2019; Davis et al., 2019b). In addition, although these methods provide anonymity to the respondents, they may still be wary of the methods and thus prevalence estimates obtained may be more conservative than the true prevalence of the behaviour (Nuno \& St John, 2015).

The nominative technique is simple to implement and appears to be free from regional/country contextual challenges, such as an unwillingness to participate in what could be perceived as gambling or games, which can occur with other specialized questioning technique methods that use randomizing devices such as dice (e.g. the randomized response technique; Davis et al., 2019a). However, if it is feasible to obtain large sample sizes, other specialized questioning techniques may be more robust for overcoming variability (e.g. the ballot box method; Bova et al., 2018). Although the nominative technique seems to be less effective for generating prevalence estimates when a behaviour is not sensitive, there is still potential for this technique to be incorporated into qualitative conservation social science methods such as informal or semi-structured interviews. Additionally, the nominative technique provides greater understanding of social group effects, unlike other specialized questioning technique methods, although our supposition that the nominative technique may provide insights into networks of users could be flawed because of the general complexity of social networks (Lansing \& Downey, 2011).

Wildlife trade research is a challenging and dynamic field that requires advancement in techniques. Accurate measures of the prevalence of wildlife consumption have long been a challenge, but our study demonstrates the feasibility of a simple shift in methods away from sometimes archaic estimates of prevalence.

Acknowledgements We thank Free the Bears and the Luang Prabang Provincial Agriculture and Forestry Office for supporting this research; Luang Prabang Lao Women's Union for providing one of the interpreters; the interpreters Boua and Amber for their support and guidance; Senagloun Vongsay (Tak), Free the Bears, for facilitating permit acquisition; J. Stacy-Dawes for creating the map figure; two anonymous reviewers and the Editor for their comments; $M$. Reinbold for assisting with proofreading; and M. Gibson for guidance and support.

Author contributions Study design: all authors; fieldwork: EOD; data analysis, writing: EOD; editing: all authors.

\section{Conflicts of interest None.}

Ethical standards This research was conducted in compliance with the principles and obligations to human subjects detailed in the ethical guidelines of the Miami University of Ohio Institutional Review Board (Protocol Number FWA00023676) and the University of Bristol Ethics Board, and otherwise abided by the Oryx guidelines on ethical standards.

\section{References}

Bova, C.S., Aswani, S., Farthing, M.W. \& Potts, W.M. (2018) Limitations of the random response technique and a call to implement the ballot box method for estimating recreational angler compliance using surveys. Fisheries Research, 208, 34-41. 
Chuang, E., Dupas, P., Huillery, E. \& Seban, J. (2019) Sex, lies, and measurement: do indirect response survey methods work? web.stanford.edu/ pdupas/CDHS_measurement.pdf [accessed 20 April 2020].

Davis, E.O., Crudge, B., Lim, T., O’Connor, D., Roth, V., Hunt, M. \& GLIKMAN, J.A. (2019a) Understanding the prevalence of bear part consumption in Cambodia: a comparison of specialized questioning techniques. PLOS ONE, 14, eo211544.

Davis, E.O. \& Glikman, J.A. (2020) An assessment of wildlife use by northern Laos nationals. Animals, 10, 685 .

Davis, E.O., Glikman, J.A., Crudge, B., Dang, V., Willemsen, M., NGuyen, T. et al. (2019b) Consumer demand and traditional medicine prescription of bear products in Vietnam. Biological Conservation, 235, 119-127.

Davis, E.O., O'Connor, D., Crudge, B., Carignan, A., Glikman, J.A., Browne-Nuñez, C. \& Hunt, M. (2016) Understanding public perceptions and motivations around bear part use: a study in northern Lao PDR of attitudes of Chinese tourists and Lao PDR nationals. Biological Conservation, 203, 282-289.

Gomez, L. \& Shepherd, C.R. (2018) Trade in bears in Lao PDR with observations from market surveys and seizure data. Global Ecology and Conservation, 15, eoo415.
Hernandez-Castro, J. \& Roberts, D.L. (2015) Automatic detection of potentially illegal online sales of elephant ivory via data mining. PeerJ Computer Science, 1, e1o.

Hinsley, A., Keane, A., St John, F.A.V., Ibbett, H. \& Nuno, A. (2019) Asking sensitive questions using the unmatched count technique: applications and guidelines for conservation. Methods in Ecology and Evolution, 10, 308-319.

LANSING, J.S. \& Downey, S.S. (2011) Complexity and anthropology. In Philosophy of Complex Systems (eds D.M. Gabbay, P. Thagard \& J. Woods), pp. 569-601. North Holland, Amsterdam, The Netherlands.

Miller, J.D. (1985) The nominative technique: a new method of estimating heroin prevalence. NIDA Research Monograph, 54, 104-24.

Nuno, A. \& S Tohn, F.A.V. (2015) How to ask sensitive questions in conservation: a review of specialized questioning techniques. Biological Conservation, 189, 5-15.

R Core Team (2018) R: A Language and Environment for Statistical Computing. R Foundation for Statistical Computing, Vienna, Austria.

St John, F.A.V., Edwards-Jones, G., Gibbons, J.M. \& Jones, J.P.G. (2010) Testing novel methods for assessing rule breaking in conservation. Biological Conservation, 143, 1025-1030. 